\title{
PLAN DE GESTIÓN PARA EL CONJUNTO CONVENTUAL DE SAN FRANCISCO DE QUITO, ECUADOR
}

Alcira Sandoval Ruiz.

Oficina de UNESCO en Quito y Representación para Bolivia, Colombia, Ecuador y Venezuela

\section{Resumen}

Son varios los motivos históricos para la intervención del convento de San Francisco. Quito fue la primera ciudad latinoamericana en convertirse en Patrimonio Mundial. Consta de 320 hectáreas arquitectónicas. El convento tiene más de $5000 \mathrm{~m} 2$ y su biblioteca cuenta con más de 4700 libros en su fondo histórico. Con el fin de dar documentación y efectuar el análisis de la restauración, donde consta descripción, significado e importancia del conjunto conventual, antecedentes del proyecto y metodología utilizada. Diagnósticos necesarios para ofrecer un turismo sostenible. Se cuenta con un Comité Ejecutivo y la gestión de recursos se realiza a través de un fideicomiso. Para terminar, el plan de gestión aspira a conservar los valores a través del tiempo.

Palabras clave: Documentación, restauración, biblioteca, metodología, proyecto.

\begin{abstract}
The historical reasons are several for the intervention of the Convent of San Francisco, Quito being the first Latin American city to become World Heritage. Architectural consists of 320 hectares, the convent has more than $5000 \mathrm{~m} 2$, and the library has over 4,700 books in its historical background. In order to begin in February 2013, a series of meetings were held, gathering documentation and analysis of restoration, which includes description, meaning and significance of the Conventual Set Project Background and methodology, diagnostics in order to sustainable tourism. This management has an Executive Committee and resource management through a trust. To complete the management plan aims to preserve the values over time.
\end{abstract}

Keywords: documentation, restoration, library, methodology, project. 
El rico patrimonio cultural del Conjunto Conventual de San Francisco es investigado, conservado, protegido y potenciado para el beneficio de la comunidad en su conjunto.

Con la puesta en marcha del plan de gestión se busca que la conservación y la gestión del Conjunto Conventual contribuya al desarrollo equilibrado y sostenible y al fortalecimiento de los valores e identidad de diversos grupos.

El sistema dará también la pauta para que la gestión y conservación del patrimonio se dé de manera ordenada, con una administración participativa, transparente y eficaz y con la actuación coordinada entre los sectores públicos y privados ${ }^{167}$.

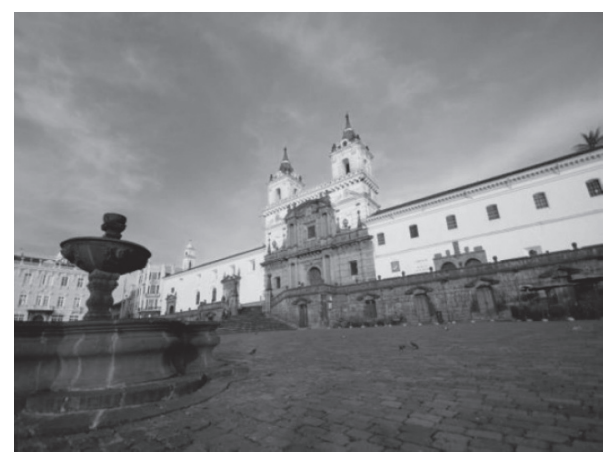

La elaboración del Plan de Gestión del Conjunto Conventual de San Francisco, constituyó para la Oficina de UNESCO en Quito y Representación para Bolivia, Colombia, Ecuador y Venezuela, la implementación de una de las actividades más importantes para la conservación y preservación de un monumento o sitio: la elaboración y puesta en marcha de su plan de gestión. Este proyecto fue realidad gracias al generoso aporte de los Fondos Fiduciarios Italianos para el Patrimonio Mundial ${ }^{168}$ y a la voluntad de cooperación de la Orden franciscana, las instituciones nacionales y locales, la sociedad civil y entes privados.

En los últimos años, los planes de gestión han cobrado especial relevancia, el Comité del Patrimonio Mundial los exige como requisito indispensable para la nominación de nuevos sitios a la Lista del Patrimonio Mundial; y las Declaratorias de Valor Universal Excepcional ${ }^{169}$ incluyen la gestión como uno de los componentes principales en su descripción, además de los criterios de inscripción y los valores de autenticidad e integridad.

Los planes de gestión son una de las herramientas más útiles y efectivas para la gestión sostenible de los sitios o sus monumentos.

\section{¿Por qué San Francisco?}

La vinculación del Conjunto Conventual de San Francisco con la Convención de 1972 de la UNESCO sobre la protección del patrimonio mundial, cultural y natural se da a partir de la inclusión de la ciudad de Quito, específicamente su centro histórico, en la Lista del Patrimonio Mundial.

167 Visión sobre el Conjunto conventual de San Francisco de Quito, elaborada en base a los objetivos y lñineamientos del plan de gestión, atributos y cualidades del mismo.

168 Los Fondos Fiduciarios Italianos tienen por objetivo reforzar la efectividad de la Convención del Patrimonio Mundial, promover la Estrategia Global para una Lista más representativa y mejorar la gestión de los sitios culturales y naturales inscritos en ella.

169 Párrafos 49 a 53 y 79 a 119 de las Directrices Prácticas para la aplicación de la Convención del Patrimonio Mundial. 
La ciudad de Quito fue el primer centro histórico de América Latina inscrito en la Lista en 1978 bajo los criterios (ii) y (iv).

\begin{tabular}{|c|c|}
\hline $\begin{array}{l}\text { Criterios de acuerdo a las Directrices Prácticas } \\
\text { de la Convención }\end{array}$ & $\begin{array}{l}\text { Justificación de los criterios de } \\
\text { acuerdo a la declaración de valor } \\
\text { universal excepcional. }\end{array}$ \\
\hline $\begin{array}{l}\text { (ii) atestiguar un intercambio de valores } \\
\text { humanos considerable, durante un periodo } \\
\text { concreto o en un área cultural del mundo } \\
\text { determinada, en los ámbitos de la arquitectura } \\
\text { o la tecnología, las artes monumentales, la } \\
\text { planificación urbana o la creación de paisajes; }\end{array}$ & $\begin{array}{l}\text { (ii) Por la influencia que la escuela } \\
\text { barroca de Quito (Escuela Quiteña) } \\
\text { tuvo en todas las ciudades de la } \\
\text { Audiencia de Quito y en Audiencias } \\
\text { vecinas, especialmente en el ámbito } \\
\text { cultural a través de la arquitectura, la } \\
\text { escultura y la pintura. }\end{array}$ \\
\hline $\begin{array}{l}\text { (iv) ser un ejemplo eminentemente } \\
\text { representativo de un tipo de construcción o de } \\
\text { conjunto arquitectónico o tecnológico, o de } \\
\text { paisaje que ilustre uno o varios periodos } \\
\text { significativos de la historia humana; }\end{array}$ & $\begin{array}{l}\text { (iv) Quito forma un conjunto armonioso } \\
\text { donde las acciones del hombre y la } \\
\text { naturaleza se fusionan creando una } \\
\text { obra única y trascendente en su } \\
\text { género. }\end{array}$ \\
\hline
\end{tabular}

La ciudad fue fundada en el siglo XVI sobre los restos de una ciudad inca y hoy en día es, uno de los centros históricos mejor conservados de América Latina. El área inscrita abarca 320 hectáreas y contiene un número importante de conventos y edificios religiosos con obras de la Escuela Barroca de Quito (Escuela Quiteña). A pesar encontrarse en un entorno topográfico muy complejo, el conjunto urbano del Centro Histórico posee una unidad estilística y volumétrica delimitada por la traza urbana de la ciudad con vías principales y plazas principales y secundarias que se mantienen casi intactas. Al interior de esta traza urbana en damero, alineada sobre los cuatro puntos cardinales, se encuentran los distintos conventos e iglesias así como casas particulares que combinan lo monumental con lo austero y que se organizan en torno a patios interiores. A pesar del paso de los años y el desarrollo, la ciudad de Quito mantiene hasta el presente la unidad y armonía en su estructura urbana ${ }^{170}$.

El Conjunto Conventual de San Francisco es uno de los monumentos más significativos del Centro Histórico de Quito. La extensión y la calidad del arte en su interior, con más de 3500 obras de arte colonial, lo hacen un ejemplo representativo de la Escuela Barroca de Quito, una de las escuelas artísticas más importantes durante el virreinato que fusiona estilos artísticos españoles, italianos, moriscos, flamencos e indígenas. El conjunto conventual cuenta con más de $5000 \mathrm{~m} 2$ construidos y aún quedan seis claustros de los 13 con los que contaba originalmente. Funciona desde 1535 como residencia de monjes franciscanos, como sitio de culto, educativo y como un sitio cultural y de esparcimiento. Es una parte central de la vida religiosa, cultural y social de Quito y uno de los edificios emblemáticos más visitados por turistas locales, nacionales e internacionales ${ }^{171}$.

170 Extracto de la declaración de valor universal excepcional de la Ciudad de Quito. Documentos WHC13/37.COM/8E y WHC-13/37.COM/8E.Add,34COM8E, aprobados durante la 37 Sesión del Comité del Patrimonio Mundial (Phnom Penh, Camboya. 17-27 June 2013).

171 Extracto del Plan del Gestión del Conjunto Conventual de San Francisco. Pags. 3 y 5. 
La iglesia principal de San Francisco, fue declarada basílica menor por el Papa Juan XXIII en 1963 y es uno de los atributos esenciales del Conjunto Conventual. El altar principal incluye obras importantes de la reconocida Escuela Quiteña, como la Virgen Alada o Virgen de Quito y el Jesús del Gran Poder. Además, el conjunto conventual, cuenta con una importante biblioteca que incluye más de 4700 volúmenes en su fondo histórico (1580-1830) y aproximadamente 4350 documentos históricos sobre la provincia franciscana.

Durante los últimos 20 años, tanto organismos internacionales como entidades nacionales y locales, han realizado numerosas inversiones e intervenciones para la restauración y conservación del patrimonio de San Francisco ${ }^{172}$. Sin embargo, a pesar de estos esfuerzos, era necesario contar con una herramienta de trabajo que permitiera una actuación planificada y con carácter sostenible, para garantizar la conservación y el mantenimiento del inmueble y sus colecciones muebles. Asimismo, era importante articular a la conservación con otros aspectos relacionados con el uso público del conjunto conventual, específicamente con las propuestas de desarrollo turístico, de investigación y de difusión.

\section{E1 Proceso}

Con el fin de dar inicio a la elaboración del plan de gestión, en febrero de $2013^{173}$ la Oficina de UNESCO en Quito, en colaboración con expertos nacionales e internacionales, la Orden Franciscana (especialmente la comunidad franciscana residente en el Convento), el antiguo Ministerio Coordinador de Patrimonio, el Ministerio Coordinador de Conocimiento y Talento Humano, el Ministerio de Cultura y Patrimonio, el Instituto Nacional de Patrimonio Cultural (INPC) y el Municipio del Distrito Metropolitano de Quito (a través de su Instituto Metropolitano de Patrimonio IMP y Quito Turismo), inició una serie de reuniones. El objetivo del plan es conseguir un equilibrio entre la conservación del monumento y su uso, garantizando la sostenibilidad de las acciones realizadas.

Para la elaboración del plan, se conformó un Comité Interinstitucional, compuesto por la comunidad franciscana, el INPC, el IMP y Quito Turismo, quienes trabajaron en el diseño del plan en coordinación con el Sector Cultura de la Oficina de UNESCO en Quito y los consultores nacionales e internacionales con experiencia en las siguientes áreas: Israel Zambrano - patrimonio mueble, Dora Arízaga - patrimonio inmueble, Carolina Castellano - planes de gestión y Jordi Tresserras - turismo sostenible.

La elaboración del plan tuvo como base una metodología participativa que contempló: la documentación y análisis de los diversos factores que inciden en la conservación y la gestión del conjunto conventual para desarrollar propuestas de actuación para diferentes componentes que incluyen a los bienes muebles e

172 Algunas de las intervenciones más importantes en este conjunto conventual, son aquellas llevadas entre 1983 y 2002, gracias al convenio firmado entre la Agencia Española de Cooperación Internacional para el Desarrollo (AECID) y el Instituto Nacional de Patrimonio Cultural (INPC). El Convento también cuenta con importantes intervenciones realizadas en distintas épocas por el antiguo Fondo de Salvamento (FONSAL) y el actual Instituto Metropolitano de Patrimonio (IMP) del Municipio del Distrito Metropolitano de Quito entre otras instituciones

El plan terminó de redactarse en octubre de 2014 y se hicieron ajustes en abril de 2015. 
inmuebles, al uso turístico, a la investigación y difusión y a la gestión propiamente dicha ${ }^{174}$.

El plan es también una herramienta para facilitar la acción concertada entre los diferentes sectores que tienen, injerencia sobre el Conjunto Conventual de San Francisco y para garantizar la transparencia en la ejecución de actividades y en el manejo de recursos financieros ${ }^{175}$.

\section{Estructura y componentes del plan}

El plan de gestión presenta una serie de líneas programáticas con acciones en el corto, mediano y largo plazo, indicando las prioridades en la ejecución de las mismas. Las líneas de acción del plan se insertan en los siguientes componentes:

- Conservación del Patrimonio Construido: Bienes Inmuebles.

- Conservación de Bienes Muebles: Obras de Arte.

- Turismo Sostenible: Ven y vive San Francisco.

Además existe un componente transversal de prevención y gestión de riesgos.

La definición de dichas líneas partió del análisis de varios aspectos, que permitieron estructurar y orientar las acciones del plan. Es así, que el plan se estructura en cuatro secciones principales:

1. Descripción, significado e importancia del patrimonio cultural del Conjunto Conventual de San Francisco. Esta sección considera los atributos y cualidades específicos a conservar y gestionar y que se definen de la siguiente manera:

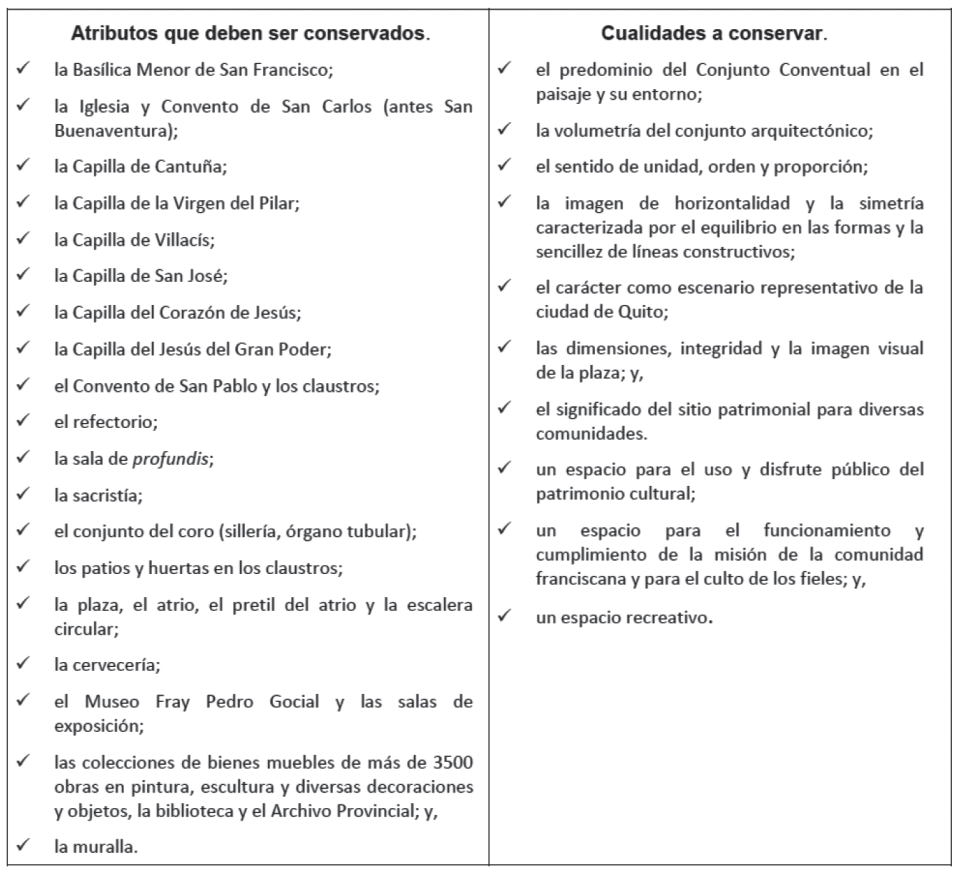

174 En Plan de gestión del Conjunto Conventual San Francisco de Quito (p. 4).

175 Idem. 
2. Antecedentes del proyecto y metodología de planificación utilizada. En esta sección se incluyen y describen las intervenciones que han tenido lugar en el conjunto conventual, así como su impacto. Se hace una descripción de cómo se llevó a cabo la construcción del plan, incluyendo las fases de planificación.

3. Diagnósticos. Los diagnósticos proporcionan una idea de la situación actual del Conjunto Conventual de San Francisco en los tres componentes antes mencionados: estado de conservación del patrimonio mueble e inmueble y las condiciones con respecto al uso turístico. Además se incluye el contexto legal y administrativo que tiene impacto directo en la gestión. Los resultados de la etapa de diagnóstico permitieron trazar las líneas de acción del plan y las actividades a realizar.

a) Patrimonio inmueble.

El diagnóstico de este componente da como resultado que las condiciones que impactan directa e indirectamente en la arquitectura del Conjunto Conventual de San Francisco responden a causas de naturaleza ambiental y antrópica que interactúan. Habiendo causas de deterioro intrínsecas (relacionadas con los materiales y técnicas constructivas), la ubicación geo-topográfica, condiciones geológicas y geodinámicas, la naturaleza del suelo y movimiento de la capa freática o bien los defectos de fábrica o construcción y en la selección de materiales.

Se indica que existe también la necesidad de un mantenimiento continuo y preventivo, así como el control de la humedad en varias áreas del convento. Otro de los factores a tomar en cuenta es la superación de la vulnerabilidad del conjunto frente a los riesgos y amenazas de tipo antrópica y vandálico con la dotación de un plan integral de seguridad y de gestión de riesgos. Se indica también que es necesario realizar la actualización de los planos arquitectónicos del convento.

b) Patrimonio mueble.

San Francisco alberga un gran conjunto de arte religioso, documentos, libros y material arqueológico ${ }^{176}$, conserva actualmente una colección que supera las $2400^{177}$ obras. Estos bienes muebles de carácter patrimonial están realizados en una gran variedad de soportes (cuero, mampostería, madera, textil, metal, piedra, cerámica, papel, entre otros). Su tipología es diversa teniendo entre las principales: decoración mural, pintura, escultura, documentos, carpintería, retablos, mobiliario, metalurgia, textil, lapidaría, etc $^{178}$. Sin contar los libros y documentos en la biblioteca histórica y el fondo documental de la provincia franciscana, que suman más de 9000 ejemplares entre ambos.

Uno de los principales desafíos para la conservación es la carencia de un plan de conservación preventiva que permita minimizar los efectos de los factores de deterioro, así como la necesidad de implementar un taller para la restauración y conservación de las obras de arte y la construcción de un espacio adecuado de reservas para los bienes muebles.

176 Parte de este material arqueológico procede de las distintas investigaciones arqueológicas ejecutadas en el convento.

177 Dato tomado del sistema ÁBACO el 02 de septiembre de 2013. Se contabilizan sólo las fichas de inventario de bienes muebles. Este dato no incluye la colección de la biblioteca, los documentos del archivo provincial y el material arqueológico.

Plan de gestión, pág. 18 
Las acciones a realizarse para superar estas dificultades, se encuentran enmarcadas en cuatro líneas de acción: conservación preventiva, conservación, conservación-restauración y museología-museografía.

c) Turismo sostenible

Para el diagnóstico de este componente se realizó el diagnóstico de la cantidad de visitantes tanto a nivel nacional, local y por monumentos; así como un análisis del potencial del conjunto conventual y las principales actividades que pueden atraer a un mayor número de turistas y se incluyó un análisis de público, con el fin de orientar la propuesta de turismo sostenible. Con los proyectos de esta línea se busca ofrecer una experiencia personal significativa y memorable desde los valores de la espiritualidad franciscana en el siglo XXI, haciendo uso de los atractivos religiosos, culturales y patrimoniales del Conjunto Conventual de San Francisco.

Algunos de los proyectos incluyen la valorización y promoción de fiestas del calendario litúrgico, fortalecimiento y mejora de visitas culturales y turísticas y planteamiento de nuevas propuestas; fortalecimiento de actividades para el público escolar y familiar; cervecería artesanal franciscana; turismo religioso en Quito y articulación del Conjunto Conventual de San Francisco; realización de estudios de capacidad de carga turística; eventos de formación en turismo religioso y especializados, mejoramiento de recorridos y uso de las TIC entre otros.

d) Propuesta para el plan de gestión.

En este apartado se incluye el marco de referencia general, las premisas del sistema, las políticas definidas, la propuesta de zonificación, líneas programáticas de actuación, la matriz de proyectos y la administración y operación del sistema de gestión.

En lo referente al marco de referencia general, sistema de gestión propuesto se inserta en el contexto legal, administrativo e institucional del Ecuador, y de Quito en particular, y considera además las diversas políticas vigentes en el nivel nacional y local con respecto al patrimonio cultural. De la misma manera, dada la categoría del Centro Histórico de Quito como Patrimonio Mundial, el sistema de gestión considera el cumplimiento de las Directrices Prácticas (2013) para la Implementación de la Convención de Patrimonio Mundial de la UNESCO de $1972^{179}$.

La zonificación se dividió en cuatro grandes rubros: los educativos, los religiosos, los de la vida monacal y los de servicios culturales o turísticos.

Las líneas programáticas agrupan a una serie de proyectos definidos durante la etapa de diagnóstico y discusión, que se articulan en tres grandes campos de acción: la conservación de bienes inmuebles, la conservación de bienes muebles y los usos educativos, culturales y turísticos.

Como eje transversal, se consideran además estrategias vinculadas con la investigación, la difusión y la promoción de proyectos de índole turística o para la habilitación de espacios para nuevos usos. 
El sistema o modelo de gestión elegido es público-privado ${ }^{180}$. Se espera que el plan de gestión sea no sólo una herramienta para conservar el Conjunto Conventual de San Francisco, sino también para gestionar la colaboración entre distintos grupos de interés en los sectores públicos y privados, para generar una mayor participación en las tareas del patrimonio y para evitar duplicidad de esfuerzos y la ejecución de acciones no planificadas en detrimento de este importante lugar patrimonial.

Se propone como una herramienta para facilitar la acción concertada entre los distintos actores de ambos sectores, que tienen interés e injerencia en San Francisco. De esta manera, se pretende que con el funcionamiento de un sistema de gestión público-privado y la ejecución de actividades conforme propuesto se garantice la transparencia en el uso de recursos, que evite la duplicidad de esfuerzos y la implementación de acciones no planificadas que vayan en detrimento de la conservación del Conjunto Conventual de San Francisco en el largo plazo.

Para poder evaluar la efectividad de la gestión, se han considerado indicadores que permitan medir el avance, logros y resultados e identificar las fallas, de tal forma que el sistema de gestión siempre esté en posibilidades de anticipar y manejar cambios, de tener capacidad de respuesta, y que por ende, sea sostenible.

Sin embargo, para que el sistema pueda funcionar, es necesario recalcar que las diversas instancias involucradas estén dispuestas a compartir la gestión con otros actores y abrir la gestión hacia niveles participativos que involucren toma de decisiones y ejecución de acciones pre acordadas, convocando a los actores que dispongan de competencias reales para hacer viable esta ejecución.

El sistema de gestión se estructura de la siguiente manera.

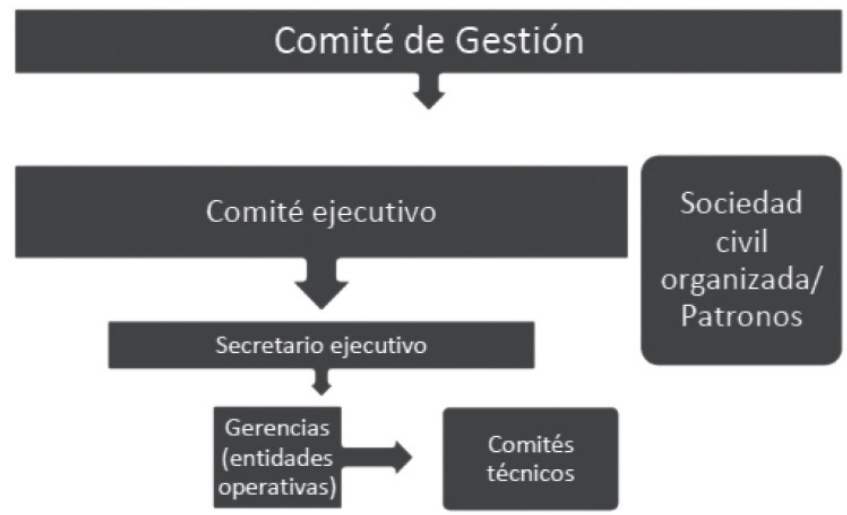

El plan describe la función y roles detallados de cada uno de ellos, entre los cuales cabe destacar al Comité de Gestión, el Comité Ejecutivo y la gestión de recursos a través de un Fideicomiso.

- Comité de Gestión. Es la asamblea de los principales actores involucrados y tiene la obligación de asegurar la ejecución del plan de gestión y actúa

180 Plan de Gestión del Conjunto Conventual de San Francisco. 
a través de su Comité Ejecutivo. El Comité de Gestión no se superpone a las instituciones competentes de la gestión del patrimonio ni a la estructura de gestión propia de la comunidad franciscana sino que, por el contrario, fortalece y potencia su capacidad de actuación. Está conformado por un representante de las siguientes instituciones: Ministerio Coordinador de Conocimiento y Talento Humano, Ministerio de Cultura y Patrimonio, Municipio del Distrito Metropolitano de Quito, Arquidiócesis de Quito, Vicaría Provincial de San Francisco, Guardianía del Convento Máximo de San Francisco.

- Comité Ejecutivo. Es el organismo de dirección permanente del Comité de Gestión. Está integrado por siete miembros permanentes representantes técnicos de las siguientes entidades: Instituto Nacional de Patrimonio Cultural, Instituto Metropolitano de Patrimonio, Empresa Pública Metropolitana de Gestión Quito Turismo, 3 representantes de la comunidad franciscana.

Sus principales funciones son conducir la implementación del Plan de Gestión y la ejecución de las decisiones del Comité de Gestión, por tanto es el ente administrador del sistema de gestión. Será también responsable de designar a la Dirección Ejecutiva.

- Gestión de recursos. Es otro factor clave para alcanzar una conservación sostenible, particularmente si se considera que el reconocimiento de la importancia del Conjunto Conventual posibilita la obtención de recursos a partir de organismos de financiamiento o por la sociedad civil. Para tal efecto, se ha creado en octubre de 2014 un Fideicomiso Mercantil de Administración de Flujos y Fuentes de Pago, denominado Fideicomiso Convento San Francisco de Quito con una cuenta corriente en el Banco Pichincha No 2100082751.

El fideicomiso permitirá al sistema de gestión, obtener recursos financieros para la realización de trabajos de conservación y preservación del Conjunto Conventual y a la vez garantizar y demostrar la transparencia en la gestión de recursos financieros.

\section{Para terminar}

El plan de gestión aspira a conservar y preservar en el tiempo los valores y atributos que han hecho del Conjunto Conventual de San Francisco lo que es:

"San Francisco es aún hoy en día un escenario representativo de la ciudad de Quito y ha funcionado no sólo como un espacio religioso por excelencia sino también como espacio para el disfrute, el encuentro y la recreación cultural y social. Su espacio abierto facilita la existencia de diversos usos y se espera que continúe siendo un eje de identidad y pertenencia para la población en su conjunto" ${ }^{181}$.

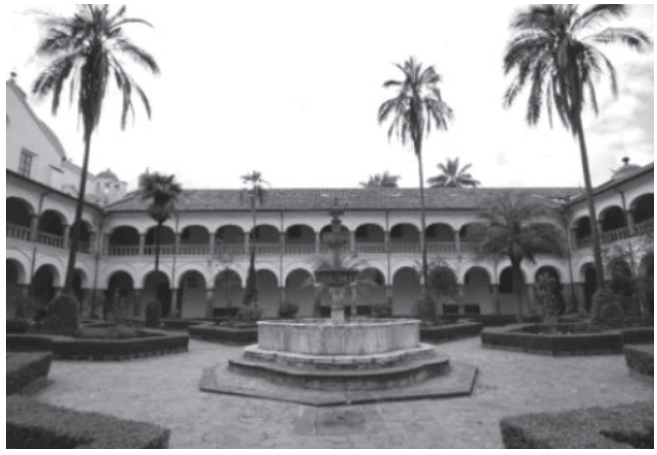

Claustro principal de San Francisco. CUNESCO O. Arregui 
En este primer año de ejecución del plan, se han obtenido resultados que motivan a seguir trabajando en la implementación del mismo, con un promedio de un $45 \%$ de avance en las actividades planificadas para los primeros 5 años.

Al mismo tiempo, es necesario destacar que el proceso para la realización del plan de gestión no habría sido posible sin la participación decidida y desinteresada de las diversas instituciones públicas y privadas, organizaciones no gubernamentales, especialistas en diversos sectores y de la comunidad relacionada con el lugar que participaron del proceso. Especialmente el INPC, Municipalidad del Distrito Metropolitano de Quito, a través del IMP y la Empresa Pública Metropolitana de Gestión de Destino Turístico - Quito Turismo, y la Comunidad Franciscana.

No podemos dejar de mencionar la importante colaboración de la Cooperación Italiana al Desarrollo y, en especial, de la Embajada de Italia en Ecuador en la persona de su Embajador, el Sr. Gianni Piccato, quienes con su valiosa gestión y apoyo, y en estrecha colaboración con la Oficina de UNESCO en Quito, lograron que esta iniciativa fuera posible.

Finalmente, la colaboración del antiguo Ministerio Coordinador de Patrimonio, los Ministerios de Cultura y Patrimonio y Turismo, la Arquidiócesis Metropolitana de Quito, de la Embajada de los Estados Unidos Mexicanos, de instituciones religiosas del Centro Histórico, de la Universidad Técnológica Equinoccial (con quien se suscribió un importante convenio de colaboración técnica), de diversas organizaciones no gubernamentales y de la sociedad civil organizada que apoyaron el proceso, cada uno desde su ámbito de acción.

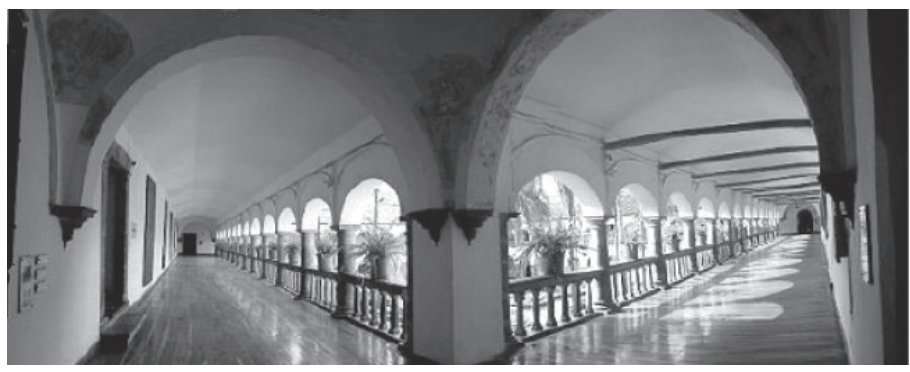

Galería superior del claustro principal. (C) UNESCO/Omar Arrregui

\section{Equipo de trabajo}

Ex Ministerio Coordinador del Patrimonio: María Belén Moncayo, ministra; Joaquín Moscoso, asesor ministerial.

Ministerio Coordinador de Conocimiento y Talento Humano: Guillaume Long, ministro.

Ministerio de Cultura y Patrimonio: Juan Diego Badillo, director de conservación y riesgo de la Subsecretaría de Patrimonio Cultural; Gabriela Eljuri, ex subsecretaria de Patrimonio Cultural; Fabián Bedón, ex asesor.

Instituto Nacional de Patrimonio Cultural: Lucía Chiriboga, directora; Ruth Aguirre, directora de Conservación; Leonardo Arcos, técnico de la Dirección de conservación; Inés Pazmiño, ex directora. 
Convento de San Francisco: Fray Mario Liroy Ortega, provincial de la Orden de San Francisco, Fray Walter Verdezoto, guardián del Convento Máximo de San Francisco de Quito, Fray Patricio Bonilla, responsable de asuntos legales del Convento Máximo de San Francisco de Quito.

Oficina de UNESCO en Quito: Alcira Sandoval Ruiz, especialista responsable del Sector Cultura; Inés Binder, asistente de programas; Beatriz Latorre, asistente.

Consultores: Dora Arízaga, responsable de patrimonio inmueble; Carolina Castellanos, responsable de gestión; Jordi Tresserras Juan, responsable de turismo sostenible; Israel Zambrano, responsable de patrimonio mueble.

Universidad Tecnológica Equinoccial: José Julio Ceballos Gómez, rector; Julio Benítez, coordinador académico de la carrera de Restauración y Museología; Katherine Basantes, Patricia Rodríguez, Patricio Corella y Yajaira Chávez, pasantes; Juan Carlos Fernández, ex coordinador académico.

\section{Información y contacto:}

Oficina de UNESCO en Quito. Representación para Bolivia, Colombia, Ecuador y Venezuela. Gral. Ignacio de Veintimilla E9-53, Quito, Ecuador. Teléfonos: (+593 2) 2528911 o 2529085. cultura-quito@unesco.org - www.unesco.org/quito.

\section{Bibliografía}

Centro del Patrimonio Mundial de la UNESCO. http:/ / whc.unesco.org/en/list/2 Expediente de Candidatura de la Ciudad de Quito a la Lista del Patrimonio Mundial. 1978

Declaración de Valor Universal Excepcional. Documentos WHC-13/37. $\mathrm{COM} / 8 \mathrm{E}$ and WHC-13/37.COM/8E.Add

Plan de Gestión del Conjunto Conventual de San Francisco. Unesco Quito y Representación para Bolivia Colombia, Ecuador y Venezuela.

Albornoz, X. E. (2009). Historia y leyenda del arte quiteño. Su iconología. Ed. Trama. Quito.

Arízaga, D. (2002). El centro bistórico de Quito: una rápida mirada a los procesos de conservación y rehabilitación y a su financiamiento. Disponible en: http://www.wmf. org/html/PDF/Arizaga_Dora.pdf [último acceso: 6.9.2013].

Breccia, G., y Fugaro, D. (2009). Catálogo informatizado del fondo biliográfico antiguo: Biblioteca del Convento Máximo de San Francisco de Quito. UL e IILA.

Buró de Convenciones de Quito (2013). Quito. Destino ideal. Quito.

Centro del Patrimonio Mundial de la UNESCO. http://whc.unesco.org/en/ list $/ 2$.

Chías J. (Dir.) (2007). Plan Q 2012. Plan estratégico de turismo de Quito. Informe fases 0 - I y II. Noviembre 2007. Chías Marketing Systems. Barcelona. 
City of Quito, Ecuador (1978) "Statement of Outstanding Universal Value, Decision WHC-13/37.COM/8E", 37th Session of the World Heritage Committee (Phnom Penh, 2013).

Declaración de Valor Universal Excepcional. Documentos WHC-13/37.COM/8E and WHC-13/37.COM/8E.Add

Del Hierro Padilla N.A. (2012). Estudio del comportamiento espacial del turista extranjero en el centro histórico de Quito como una herramienta para la mejora de la señalética, movilidad y seguridad turística. Tesis de grado en Ingeniería Administración de Empresas Turísticas de la Facultad de Turismo especialización Hotelería. Universidad del Pacífico. Junio 2012. Quito. http://dspace.upacifico.edu.ec/jspui/bitstream/123456789/142/1/60503.pdf [último acceso: 7.9.2013].

Del Pino, I. (2010). Centro bistórico de Quito, una centralidad urbana hacia el turismo. FLASCO - Sede Ecuador / Abya Yala. Quito.

Del Pino, I. (2012). "El Centro Histórico de Quito y el Turismo Cultural”, en Revista América Patrimonio 4. Disponible en http:/ / www.revistaamericapatrimonio.org/art_3_4.pdf [último acceso 30/08/2013].

EPMGDT - Quito Turismo (2012a). Plan Estratégico. Empresa Pública Metropolitana de Gestión de Destino Turístico Quito Turismo 2012-2015. Quito.

EPMGDT-Quito Turismo (2012b). Caracterización del turismo receptor en el DMQ. N². Septiembre 2012. Distrito Metropolitano de Quito. Quito.

EPMGDT - Quito Turismo (2012c). Perfil del turista de negocios y su gasto promedio. Quito Turismo. Quito.

EPMGDT - Quito Turismo (2013). La experiencia del destino turístico. Quito en cifras. Quito Turismo. Abril 2013. Quito.

Expediente de Candidatura de la Ciudad de Quito a la Lista del Patrimonio Mundial. 1978.

Fernández, L. (1999). Museología y museografía. Barcelona: Serbal.

Fonsal (2010). Proyecto Rehabilitación integral Iglesia y Convento de San Francisco (plano digital). Quito: Fonsal.

Galeotti, M. (2006). Estudio Integral de los bienes muebles de la Iglesia de San Francisco. Memoria técnica: Diagnóstico y anteproyecto (inédito). Quito: Fonsal.

INPC/AECID (2011). Iglesia y Convento San Francisco, una historia para el futuro. Quito.

INPC. (2011). Instructivo para fichas de registro e inventario. Bienes muebles. Quito: INPC.

IPCE. (2011). Plan Nacional de Conservación Preventiva. Madrid: IPCE.

Licciardi G. \& Amirtahmasebi R. (Eds.) (2012). The economy of uniqueness. Investing in historic city cores and cultural heritage assets for sustainable development. Urban Development Series. Washington DC: World Bank.

Matiz, P. y Ovalle, B. (2006). Conservación preventiva en museos: evaluación de riesgos. Bogotá: Universidad Externado de Colombia. 
Mercé, J. y Gallegos, J. (2011). San Francisco una historia para el futuro. Quito: Instituto Nacional de Patrimonio Cultural, Escuela Taller San Andrés, Programa de Preservación del Patrimonio para el Desarrollo. AECID.

Mercé, J. y Silva, M. (2004). Plan director de restauración. Convento e iglesia de San Francisco. Volumen II y III. Quito: Instituto Nacional de Patrimonio Cultural, Embajada de España en Ecuador, Cooperación Española.

Ministerio de Turismo (2007). Plan estratégico de desarrollo del turismo sostenible en Ecuador hacia el año 2020. PLANDETUR 2020. Quito: Ministerio de Turismo y Banco Interamericano de Desarrollo.

Ministerio de Turismo (2009a). Plan Integral de Marketing Turístico de Ecuador (internacional)- PIMTE 2014. Quito: Ministerio de Turismo.

Ministerio de Turismo (2009b). Plan Integral de Marketing Turístico para el Turismo Interno de Ecuador - PIMTE 2014. Quito: Ministerio de Turismo.

Municipio del Distrito Metropolitano de Quito (1999). Plan Estratégico 1999-2005. Quito.

Municipio del Distrito Metropolitano de (2003).- Centro histórico de Quito: plan especial. Quito: Municipio del Distrito Metropolitano de Quito y Junta de Andalucía.

Municipio del Distrito Metropolitano de Quito (2004a). Plan Bicentenario. Plan de Gobierno 2005-2009. Quito.

Municipio del Distrito Metropolitano de Quito (2004b). Plan Equinoccio 21 - Quito hacia el 2025. Quito.

Municipio del Distrito Metropolitano de Quito (2008). Quito, productiva y competitiva. Vol 2. Quito.

Municipio del Distrito Metropolitano de Quito (2008). Quito babitable y armónica. Eje territorial. Volumen 3. Quito.

Municipio del Distrito Metropolitano de Quito (2009). Plan de movilidad sostenible. Centro bistórico de Quito. Quito.

Municipio del Distrito Metropolitano de Quito (2012). Memoria de actividades. Quito Capital Americana de la Cultura 2011. Quito.

Plan de Gestión del Conjunto Conventual de San Francisco. Unesco Quito y Representación para Bolivia Colombia, Ecuador y Venezuela.

PUCE (1978). "Museos de Quito", en Revista de la Universidad Católica 19: 82-97. Quito: Pontificia Universidad Católica del Ecuador.

Rojas E. (2012). "Governance in historic core regeneration projects", en Licciardi, G. y Amirtahmasebi, R. (eds.). The economy of uniqueness. Investing in bistoric city cores and cultural heritage assets for sustainable development. Urban Development Series. Washington DC: World Bank. pp. 143-181.

Rojas E. y Lanzafanne, F. (Eds.) (2011). City development. The experiences of ten World Heritage Sites. Washington DC: Inter-American Development Bank. 
Webster S.V. (2012a). Quito, ciudad de maestros: arquitectos, edificios y urbanismo en el largo siglo XVII. Quito: Abya-Yala.

Webster S.V. (2012b). "La desconocida historia de la construcción de la iglesia de San Francisco en Quito" en Procesos. Revista Ecuatoriana de Historia 35 (1 Semestre 2012): 37-66. Quito. Resumen 